\title{
Regeneration Mechanism of Full Thickness Cartilage Defect Using Combination of Freeze Dried Bovine Cartilage Scaffold - Allogenic Bone Marrow Mesenchymal Stem Cells - Platelet Rich Plasma Composite (SMPC) Implantation
}

\author{
Dwikora Novembri Utomo ${ }^{1,3}$, Fedik Abdul Rantam ${ }^{2,3}$, Ferdiansyah ${ }^{1,2,3}$, \\ Purwati ${ }^{2,3}$ \\ ${ }^{1}$ Department of Orthopaedics and Traumatology, Faculty of Medicine, Airlangga University, \\ Surabaya, Indonesia \\ ${ }^{2}$ Stem Cell Research and Development Centre, Airlangga University, Surabaya, Indonesia \\ ${ }^{3}$ Cell and Tissue Bank, Dr. Soetomo General Hospital, Surabaya, Indonesia
}

Corresponding author: dwikora_utomo@yahoo.com

Keywords: regeneration mechanism, full thickness cartilage defect, scaffold, BM-MSCs, platelet rich plasma

\begin{abstract}
Cartilage defect has become serious problem for orthopaedic surgeon and patients because of its difficult healing that might occur when articular cartilage damage never reach subchondral layer. In this study, we used combination of freeze dried bovine cartilage (FDBC) scaffold, bone marrow mesenchymal stem cells (BM-MSCs), and platelet rich plasma (PRP) composite (SMPC) implanted in full thickness cartilage defect. This study is to explain its regeneration mechanism. This is true experimental research with post-test only control group design using New Zealand White Rabbit. 50 rabbits is divided into three groups of SMPC, BM-MSCs and FDBC. 37 rabbits evaluated after twelve weeks. Histopathologic examination showed the number of chondrocytes, collagen thickness and cartilage width are highest on SMPC group. Immunohistochemical examination showed SMPC group has the highest number of chondroprogenitor cells express FGF-2R, Sox-9, and MAPK. Brown Forsythe test resulted in significant increase the number of chondrocytes $(p=0,010)$, collagen thickness $(p=0,000)$, and cartilage surface width $(p=0,015)$, and increase FGF-2R $(p=0,000)$, MAPK $(p=0,000)$, and Sox-9 $(p=0,000)$ on SMPC group. Using path analysis, there is strong influence from FGF-2R, MAPK, and Sox-9 to the increase of chondrocytes, collagen thickness, and cartilage surface width. Hence, SMPC implantation mechanism of full thickness cartilage defect regeneration can be explained.
\end{abstract}

\section{Introduction}

Cartilage defect is common disease found $65 \%$ cases and more than 31,000 arthroscopic procedures performed [1]. Articular cartilage defect has become serious problem for orthopaedic surgeon and patients because of its difficult healing that might occur when articular cartilage damage never reach subchondral layer [2]. Articular cartilage has poor regeneration ability due to its avascular nature, minimal cell availability, without basal membrane and nerve innervation, therefore it depend solely on diffusion process to gain nutrition. Full thickness cartilage defect will form clot surround them due to its blood supply [3]. Several measures have been developed to solve this problem, includes drug prescription and some operative techniques but none of these give good results [4].

Autologous Chondrocytes Implantation (ACI) is implantation procedure on full thickness cartilage defect isolated and cultured in monolayer and covered by sewn periosteum. Cartilage as chondrocytes source taken from non-weight bearing joints. But it is also bring up tissue hypertrophy, layer damage, graft integration failure, and chondromalacia as consequences [5,6]. Matrix Induced Chondrocyte Implantation (MACI) is continous improvement from ACI, an applied tissue engineering that combine mesenchymal stem cells or chondrocytes, and scaffold with or 
without growth factor [7]. ACI and mosaicplasty recommended to regenerate defect in patients with cartilage defect diameter less than $2 \mathrm{~cm}^{2}$. Cartilage defect that is larger or replacement in almost the entire joint surface requires another treatment method [8].

Tissue engineering uses combination (chondrocytes or mesenchymal stem cells), scaffold and growth factors. Chondrocyte has limits include already differentiated and slow to proliferate after implanted, risk of contamination during isolation, and extraction more difficult in human [9]. Mesenchymal stem cells (MSCs) implantation will bring new hope in treatment of articular cartilage damage since their ability to differentiate as chondrocyte shall produce extra cellular matrix to regenerate cartilage defect [5]. In this study we used allogenic BM-MSCs which known has immunosuppressive effect and no Graft Versus Host Disease (GVHD) [10,11]. BM-MSCs in combination with scaffold will regenerate full thickness cartilage defect by facilitate proliferation and produce matrix as progenitor cells source that is more accessible, easily manipulated, and renew themselves [12].

Freeze Dried Bovine Cartilage Powder (FDBC) contains extracellular matrix and able to stimulate chondrogenesis such as CDMP1/GDF5 [13]. MSC-scaffold combination mixed with platelet rich plasma contains various growth factors. This composite will be implanted on rabbit's full thickness cartilage defect with diameter $4 \mathrm{~mm}^{2}$ that is equivalent to $6 \mathrm{~cm}^{2}$ lesions on adult humans. But regeneration mechanism of full thickness cartilage defect using SMPC is still unclear according to Ornitz (2005) and Hoffmann (2004) research [14,15].

The aim of this study is to explain regeneration mechanism on full thickness cartilage defect implanted with Freeze Dried Bovine Cartilage Powder Scaffold- Mesenchymal Stem Cells-Platelet Rich Plasma Composite (SMPC). For further SMPC can produced so that it can begin clinical studies in patients with full thickness cartilage defect (clinical research).

\section{Materials and Methods}

This study was true experimental research with post-test only control group design using New Zealand white rabbit as subject. Rabbits were divided into three groups (FDBC, MSC, SMPC) and evaluated after twelve weeks. Microscopic and histopatologic condition were evaluated using histopathology and immunohistochemistry.

\section{SMPC Fabrication and Characterization}

Freeze dried bovine cartilage scaffold was made through 3 stages: tissues freezing, primary drying using sublimation and secondary drying to remove remains of water. This product contains water less than 5\% and its process in accordance with Tissue Bank International.

Rabbit's BM-MSCs were cultured and characterized with immunohistochemical using monoclonal antibody FITC anti-human CD45 and FITC anti-human CD105 (Biolegend). Observation of CD45/CD105 performed using flourescence microscope. MSCs will show positive expression on CD105 in green luminescence.

Platelet rich plasma from rabbit's blood was produced using centrifugation in $3000 \mathrm{rpm}$ for 15 minutes at $4{ }^{\circ} \mathrm{C}$ twice. PRP-Gel active after mixed with $\mathrm{CaCl}_{2}$ and thrombin. Implantation of BM-MSCs was performed by mixing FDBC $20 \mu \mathrm{g}$ and PRP 80\% volume, and immersed into CCM's solution and stem cells suspension for 16 hours. MSCs attachment on FDBC-PRP was observed using Scanning Electron Microscope.

\section{Toxicity Tests}

MTT assay used to determine biomaterials toxicity (in this study is FDBC) on stem cell. MTT assay result was analyzed using one way ANOVA statistics between MSC, MSC+PRP, $\mathrm{MSC}+\mathrm{FDBC}$, and MSC+FDBC+PRP groups. 


\section{Alloreactivity Tests}

This test performed by implanting BM-MSCs on rabbit muscle. After 3 weeks postinjection, samples were measured through histopathology using hematoxyllin-eosin coloring (HE). The number of lymphocytes, monocytes, and PMN per field of view at 10 field of view was then examined.

\section{Biocompatibility Tests}

Biocompatibility performed through in-vivo in New Zealand White Rabbit. 50 rabbits is divided into three groups of SMPC, BM-MSC, and FDBC, and 37 rabbits were evaluated after 12 weeks. Histopathology was performed by calculating macrophages in muscles, while immunohistochemistry identified by IL-1, which is the most significant cytokine found.

\section{Results}

Bone marrow mesenchymal stem cells was cultured for 2 weeks to get enough cells $\left(2 \times 10^{6}\right.$ cells). The number of MSCs which is optimal for immobilization to the defect $4 \mathrm{~mm}^{2}$ is $1 \times 10^{6}$ per $\mathrm{cm}^{2}$. MSCs attachment on FDBC scaffold and PRP-Gel was observed as shown in Figure 1.

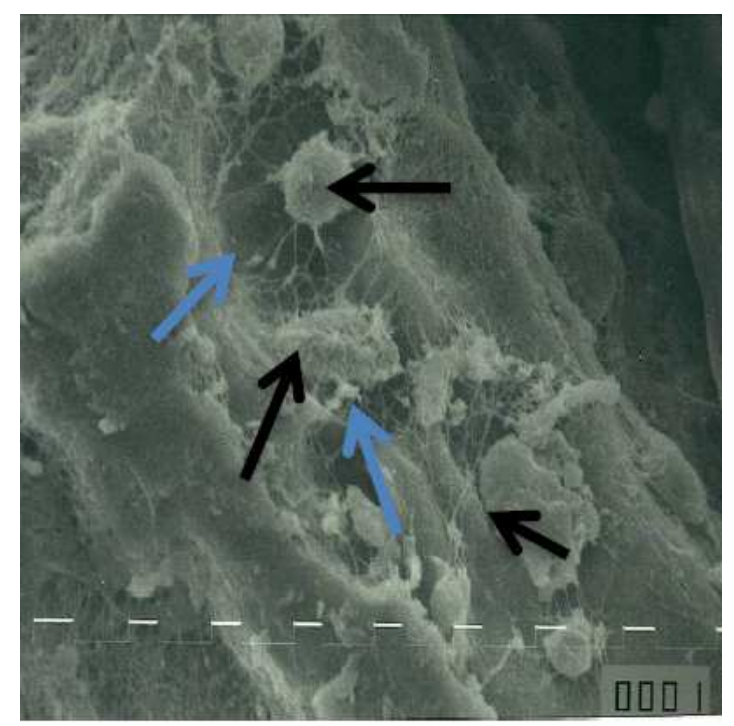

Figure 1. Seeding MSCs into FDBC-PRP using SEM magnification 2000x. Black arrow is stem cell and blue arrow is FDBC scaffold.

\section{Toxicity Tests Results}

Toxicity comparison of each material is presented in Figure 2. From one way ANOVA found no significant difference between MSC, MSC+PRP, MSC+FDBC, and MSC+FDBC+PRP $(\mathrm{p}=0,130)$. This means that FDBC is not toxic to stem cell.

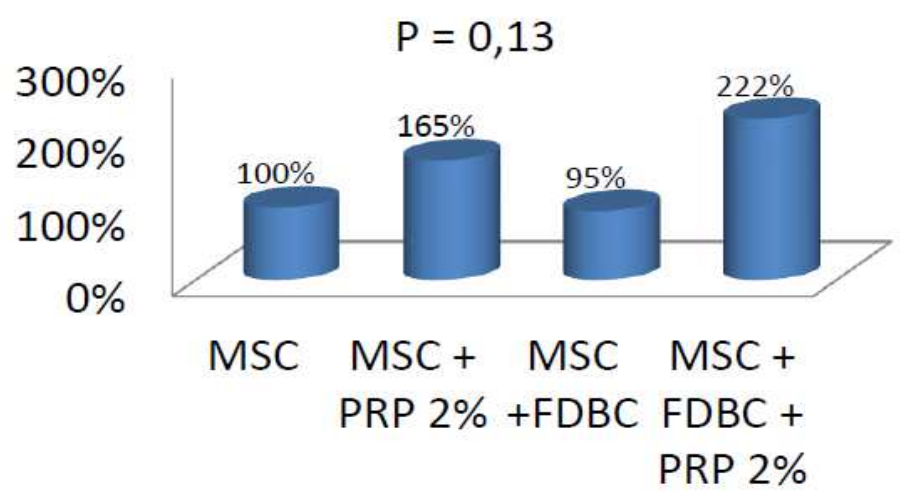

Figure 2. Toxicity test results. 


\section{Alloreactivity Test Results}

Alloreactivity analysis performed by measuring serum levels IL-10 and the number of lymphocytes, monocytes, and polimorphonuclear (PMN). Mann-Whitney analysis result showed no significant differences in serum levels IL-10 $(\mathrm{p}=0,250)$, the number of lymphocytes $(\mathrm{p}=0,130)$, monocytes $(p=0,230)$, and PMN $(p=0,340)$. Figure 3 (A and $B)$ showed distribution of inflammatory cells did not differ significantly between treatment group and control group.

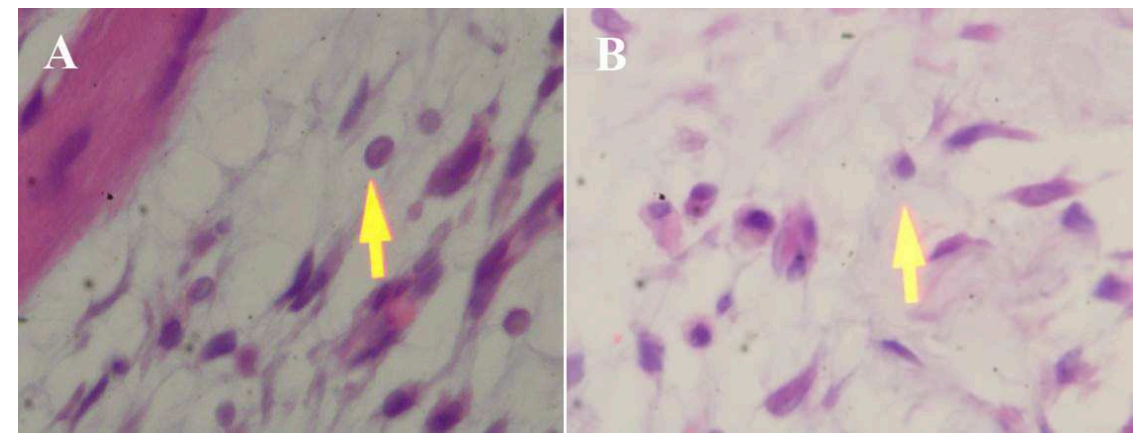

Figure 3. Histopathology of femoral muscle using HE coloring, magnification 200x. Yellow arrows showed distribution of inflammatory cells; A. Treatment group B. Control group.

\section{Biocompatibility Test Results}

Macroscopic Appearance

There is still clearly visible defect in FDBC and MSC groups. Defect in MSC almost gone even not covered all. SMPC group showed a good healing as shown in Figure 4.

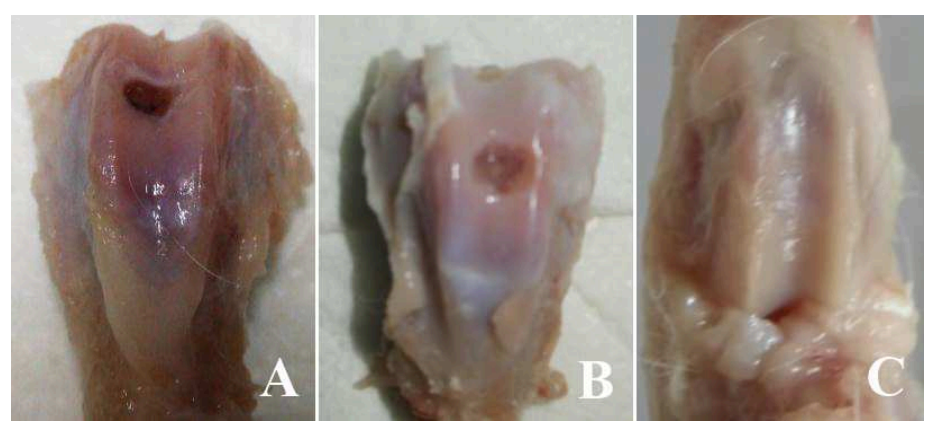

Figure 4. Macroscopic appearance in cartilage defect after 3 months. A. Implantation using FDBC; B. Implantation using MSC; C. Implantation using SMPC.

Histopathology Examination

Histopathologic examination of the number of chondrocytes and collagen thickness in each group shown in Figure 5 and Figure 6 (Supplementary Information). The highest results for the number of chondrocytes, collagen thickness, and cartilage surface width found in SMPC group. Brown-Forsythe Statistic analysis obtained $\mathrm{p}=0,010$ (Table 1), $\mathrm{p}=0,000$ (Table 2), and $\mathrm{p}=0,015$ (Table 3) which means that there are difference results between FDBC, MSC, and SMPC groups.

\section{Immunohistochemistry Examination}

Immunohistochemical examination of FGF-2R, MAPK, and Sox-9 shown in Figure 7, Figure 8, and Figure 9 (Supplementary Information). The number of condroprogenitor cells that express FGF2-R, MAPK, and Sox-9 most found in SMPC group. Brown-Forsythe Statistic analysis obtained $p=0,000$ in Table 4, Table 5 , and Table 6 which means there are significant difference results between FDBC, MSC, and SMPC groups. 
Table 1. The number of chondrocytes in three groups.

\begin{tabular}{|l|c|r|r|r|r|r|}
\hline \multirow{2}{*}{ Group } & \multirow{2}{*}{$\mathrm{n}$} & \multicolumn{4}{|c|}{ The number of chondrocytes (cells) } & \multirow{2}{*}{ Brown-Forsythe } \\
\cline { 3 - 6 } & & Average & \multicolumn{1}{c|}{ SD } & \multicolumn{1}{c|}{ Min } & \multicolumn{1}{c|}{ Max } & \multicolumn{1}{c|}{ Statistic } \\
\hline FDBC & 12 & $41054,00^{\mathrm{a}}$ & 43763,49 & 8217 & 169670 & \multirow{2}{*}{$\begin{array}{c}\text { Stat }=6,523 \\
\mathrm{p}=0,010^{*}\end{array}$} \\
\hline MSC & 12 & $127458,58^{\mathrm{b}}$ & 101519,57 & 32858 & 340638 & \\
\hline SMPC & 13 & $399885,54^{\mathrm{b}}$ & 443180,89 & 16092 & 1470701 & \\
\hline
\end{tabular}

Table 2. Collagen thickness in three groups.

\begin{tabular}{|c|c|c|c|c|c|c|}
\hline \multirow[t]{2}{*}{ Group } & \multirow[t]{2}{*}{$\mathrm{n}$} & \multicolumn{4}{|c|}{ Collagen thickness $(\mu \mathrm{m})$} & \multirow{2}{*}{$\begin{array}{c}\text { Brown-Forsythe } \\
\text { Statistic }\end{array}$} \\
\hline & & Average & SD & Min & Max & \\
\hline FDBC & 12 & $0,71^{\mathrm{a}}$ & 0,19 & 0,31 & 0,97 & \multirow{3}{*}{$\begin{array}{c}\text { Stat }=39,833 \\
\mathrm{p}=0,000^{*}\end{array}$} \\
\hline $\mathrm{MSC}$ & 12 & $1,79^{\mathrm{b}}$ & 0,51 & 0,94 & 2,89 & \\
\hline SMPC & 13 & $2,21^{b}$ & 0.52 & 1,25 & 2,83 & \\
\hline
\end{tabular}

Table 3. Cartilage surface width in three groups.

\begin{tabular}{|c|c|c|c|c|c|c|}
\hline \multirow[t]{2}{*}{ Group } & \multirow[t]{2}{*}{$\mathrm{n}$} & \multicolumn{4}{|c|}{ Cartilage surface width $\left(\mu \mathrm{m}^{2}\right)$} & \multirow{2}{*}{$\begin{array}{c}\text { Brown-Forsythe } \\
\text { Statistic }\end{array}$} \\
\hline & & Average & $\mathrm{SD}$ & Min & Max & \\
\hline FDBC & 12 & $30230,34^{\mathrm{a}}$ & 22045,88 & 5556,05 & 79463,91 & \multirow{3}{*}{$\begin{array}{c}\text { Stat }=5,850 \\
p=0,015^{*}\end{array}$} \\
\hline MSC & 12 & $147821,44^{b}$ & 118319,76 & 38207,64 & 396091,58 & \\
\hline SMPC & 13 & $399973,15^{b}$ & 470783,74 & 18714,35 & 1710118,80 & \\
\hline
\end{tabular}

Table 4. FGF-2R percentage in three groups.

\begin{tabular}{|c|c|c|c|c|c|c|}
\hline \multirow[t]{2}{*}{ Group } & \multirow[t]{2}{*}{$\mathrm{n}$} & \multicolumn{4}{|c|}{ FGF-2R (\%) } & \multirow{2}{*}{$\begin{array}{c}\text { Brown-Forsythe } \\
\text { Statistic }\end{array}$} \\
\hline & & Average & SD & Min & Max & \\
\hline FDBC & 12 & $2,58^{\mathrm{a}}$ & 1,73 & 0,94 & 6,34 & \multirow{3}{*}{$\begin{array}{c}\text { Stat }=72,106 \\
p=0,000^{*}\end{array}$} \\
\hline MSC & 12 & $11,09^{b}$ & 11,34 & 1,00 & 34,57 & \\
\hline SMPC & 13 & $42,40^{b}$ & 10,01 & 29,74 & 70,82 & \\
\hline
\end{tabular}

Table 5. MAPK percentage in three groups.

\begin{tabular}{|c|c|c|c|c|c|c|}
\hline \multirow[t]{2}{*}{ Group } & \multirow[t]{2}{*}{$\mathrm{n}$} & \multicolumn{4}{|c|}{ MAPK (\%) } & \multirow{2}{*}{$\begin{array}{c}\text { Brown-Forsythe } \\
\text { Statistic }\end{array}$} \\
\hline & & Average & SD & Min & Max & \\
\hline FDBC & 12 & $2,19^{\mathrm{a}}$ & 1,64 & 0,88 & 5,35 & \multirow{3}{*}{$\begin{array}{c}\text { Stat }=25,651 \\
\mathrm{p}=0,000^{*}\end{array}$} \\
\hline MSC & 12 & $3,60^{b}$ & 2,47 & 0,52 & 8,00 & \\
\hline SMPC & 13 & $9,87^{b}$ & 4,03 & 3,69 & 16,03 & \\
\hline
\end{tabular}

Table 6. Sox-9 percentage in three groups.

\begin{tabular}{|c|c|c|c|c|c|c|}
\hline \multirow{2}{*}{ Group } & \multirow[t]{2}{*}{$\mathrm{n}$} & \multicolumn{4}{|c|}{ Sox-9 (\%) } & \multirow{2}{*}{$\begin{array}{c}\text { Brown-Forsythe } \\
\text { Statistic }\end{array}$} \\
\hline & & Average & SD & Min & Max & \\
\hline FDBC & 12 & $3,86^{\mathrm{a}}$ & 2,11 & 1,23 & 7,84 & \multirow{3}{*}{$\begin{array}{c}\text { Stat }=29,924 \\
p=0,000^{*}\end{array}$} \\
\hline $\mathrm{MSC}$ & 12 & $5,76^{b}$ & 4,55 & 1,43 & 17,01 & \\
\hline SMPC & 13 & $22,10^{b}$ & 10,23 & 9,15 & 46,04 & \\
\hline
\end{tabular}

Description : * significant at $\alpha=0,05$

a,b different superscript shows the differences among groups (based on multiple comparisons Games-Howell) 


\section{Path Analysis of SMPC Effect}

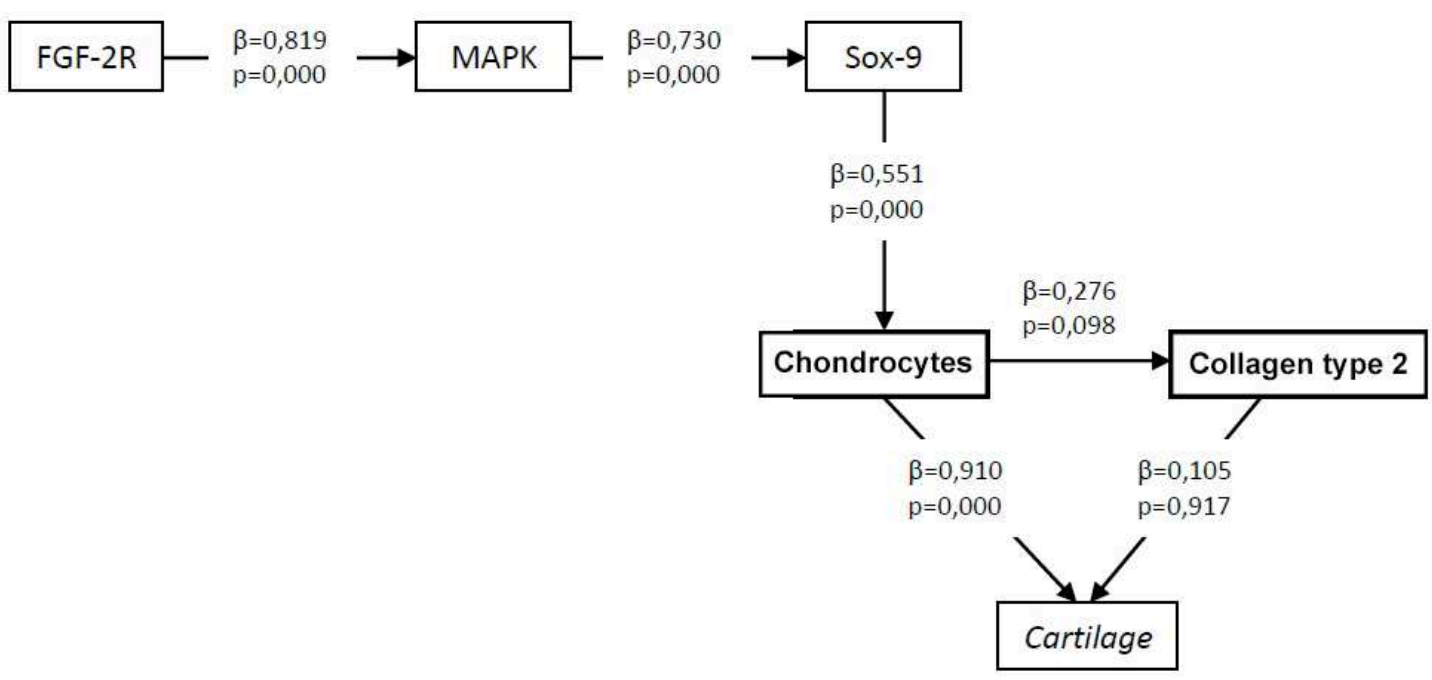

Figure 10. Path analysis of SMPC effect in regeneration of full thickness cartliage defect.

Path analysis (Figure 10) showed FGF-2R has strong influence on MAPK, MAPK on Sox9, Sox-9 on chondrocytes, chondrocytes on cartilage, and Sox-9 on FGF-2R.

\section{Discussion}

SMPC composite is able to fill defect in cartilage because it is gel so it can follow the shape of joint surface. This study found that concentration of PRP $80 \%$ volume and scaffold $20 \mu \mathrm{g}$ is ideal for MSCs growth. This is correlate with cartilage composition consists $80-90 \%$ water and $5-10 \%$ extracellular matrix [16].

\section{Regeneration Mechanism of Full Thickness Cartilage Defect}

Brown-Forsythe Statictics analysis obtained percentage of condroprogenitor cells that express FGFR-2, MAPK, and Sox9, the number of chondrocytes, and collagen thickness were significantly higher in SMPC than in MSC and FDBC groups. This may explain regeneration mechanism of full thickness cartilage defect with SMPC. This mechanism involves the role of MSCs, FDBC, and PRP. FDBC contains chondrogenesis (CDMP1/GDF5), a BMP-like molecules that can bind BMP-2-1A receptor and release growth factors during injury include TGF- $\beta$, IGF, BMP-2, BMP-7. PRP as result of blood processing has wide variety of growth factors. In another study showed an increased of BMP-2 on PRP-Gel application in animal experimental [17]. This indicates that PRP has an important role for BMP-2 which is one of main originators of chondrogenesis.

The first mechanism is BM-MSCs in SMPC which implanted in cartilage joint defect will bind bone morphogenetic protein-2 (BMP-2) derived from PRP, and BMP-2 binds CDMP-1 derived from scaffold through BMPR-1A receptor which contained BM-MSCs surface. This bond will induce an increase in expression of fibroblast growth factor-2R (FGF-2R) and fibroblast growth factor-3R (FGF-3R). If there is binding between FGF-3 and FGF-3R in BM-MSCs surface, MSCs cytosol will be increased the activity of mitogen-activated protein kinase (MAPK). Furthermore, MAPK which already activated induces T-box factor (brachyury) to conduct upregulation expression of FGF-2R and FGF-3R and stimulates Sox-9 as chondrogenic transcription factor (CTF). CTF translocated into MSCs nucleus to induce mitotic genes so that they develop into condroprogenitor cells become chondrocytes which produce extracellular matrix.

The second mechanism is FDBC scaffold contains CDMP-1/GDP-5 (growth factor that has chondrogenesis ability) will provide signaling to BM-MSCs to differentiate into condroprogenitor cells which subsequently became chondrocytes, as it also stimulate chondrocytes to produce extracellular matrix like proteoglycan and collagen, so the end result is cartilage formation. 
The third mechanism is full thickness cartilage defect will release cytokines which is important in healing process include TGF- $\beta 2$, IGF, BMP-2, BMP-7. Transforming Growth Factor beta (TGF- $\beta$ ) binds to TGF- $\beta$ R in BM-MSCs surface along with TGF- $\beta$ derived from PRP to induce MAPK as in first mechanism. Moreover, TGF- $\beta$, IGF-1, BMP-2, BMP-7 also role in regeneration of full thickness cartilage defect by stimulating chondrocytes to synthesize extracellular matrix, which produce cartilage formation. From all mechanisms and immuno-histochemistry, it can be concluded that SMPC has strong influence on growth factors.

\section{Regeneration Analysis Results}

Cartilage surface width in SMPC is bigger than FDBC and MSC group. The same thing with previous explanation that BMP-2 and BMPR-1A is important to start regeneration process of cartilage defect, so that in SMPC group, growth factors derived from scaffold, PRP, and defect cartilage will contribute more than two other groups. Another role is derived from the number of BM-MSCs that is more than FDBC group. Cartilage formation will also be more widely established in SMPC group, because BM-MSCs already proliferated and differentiated into condroprogenitor cells become chondrocytes and will be stimulated to produce extracellular matrix either from scaffold (CDMP1/GDF5) or defect (BMP-2, IGF-1, BMP-7, TGF- $\beta$ ). Chondrocytes which formed along with extracellular matrix will form cartilage wider than two other groups.

In path analysis found the number of collagen formed is not significantly correlated with cartilage formation. It can be explained that evaluation of this study was conducted at 12 weeks so regeneration of cartilage is not perfect though chondrocytes layers has been arranged, and tidemark as boundary between cartilage and subchondral bone evident at week-24, and not all condroprogenitor differentiated into chondrocytes to produce collagen [18].

\section{Comparison of SMPC Results to Other Methods}

Cartilage from SMPC group showed a good incorporation in microscopic. Scaffold as an important medium will regenerate cartilage if accompanied by optimum administration of MSCs. This proves that the defect which is given only by scaffold does not get adequate number of MSCs to repair. The same thing with MSCs only that is not good enough to repair because cells would be out of defect area due to gravity as in the ACI. This study obtained regeneration results better than Junji which is using the ACI technique, where obtained uneven cells at the sites of implantation [19].

This study obtained surface cartilage regeneration same level with normal cartilage surface on 12 weeks evaluation post-implantation. This result is better than Niswander study in rats, which is implanted BM-MSCs on full thickness cartilage defect without using scaffold in treatment group, and comparison group made defects of the same size at $2 \mathrm{~mm} \times 2,5 \mathrm{~mm} \times 1 \mathrm{~mm}$ which is given by FBS only. The results obtained cartilage regeneration was better but still there are hollows on the surface or is not level with normal cartilage [20].

Wakitani conducted a study regeneration on full thickness cartilage defect in New Zealand white rabbit by making a hole size of $6 \times 3 \times 3 \mathrm{~mm}$. In defect given treatment using implantation of BM-MSCs + collagen gel, compared to treatment of BM-MSCs and defect only. The results obtained in BM-MSCs + collagen gel and BM-MSCs did not differ significantly, but it's better than just by defect only. On 12 weeks evaluation, in BM-MSCs + collagen gel group and BM-MSCs group obtained regeneration of cartilage surface is thinner and the surfac is irregular (not flat). In this study, SMPC on 12 weeks evaluation showed better results in cartilage regeneration, which is thickness and flat (regular) [21].

The new findings in this study are: first, FDBC is still contains organic cartilage compared to hyaluronan or collagen which is currently most popular and widely used. FDBC has never been used as scaffold for cartilage regeneration. Second, SMPC both in in-vitro and in-vivo is an ideal composition for tissue engineering on full thickness cartilage defect. 


\section{Conclusion}

Regeneration mechanism of full thickness cartilage defect in SMPC is through MSC FGF-2R signals in surface which is attached to scaffold and will be forwarded to MAPK in nucleus, thus synthesize Sox-9. Protein Sox induces MSC proliferation and maturation into chondrocytes. Chondrocytes synthesize collagen type 2 but in regulated number so that regeneration of cartilage in accordance with joint cartilage.

\section{Abbreviations \\ FDBC : Freeze Dried Bovine Cartilage \\ BM-MSCs : Bone Marrow Mesenchymal Stem Cells \\ MSC : Mesenchymal Stem Cell \\ PRP : Platelet Rich Plasma \\ SMPC : FDBC Scaffold - MSC - PRP Composite}

\section{Supplementary Information}

Biocompatibility Test Results
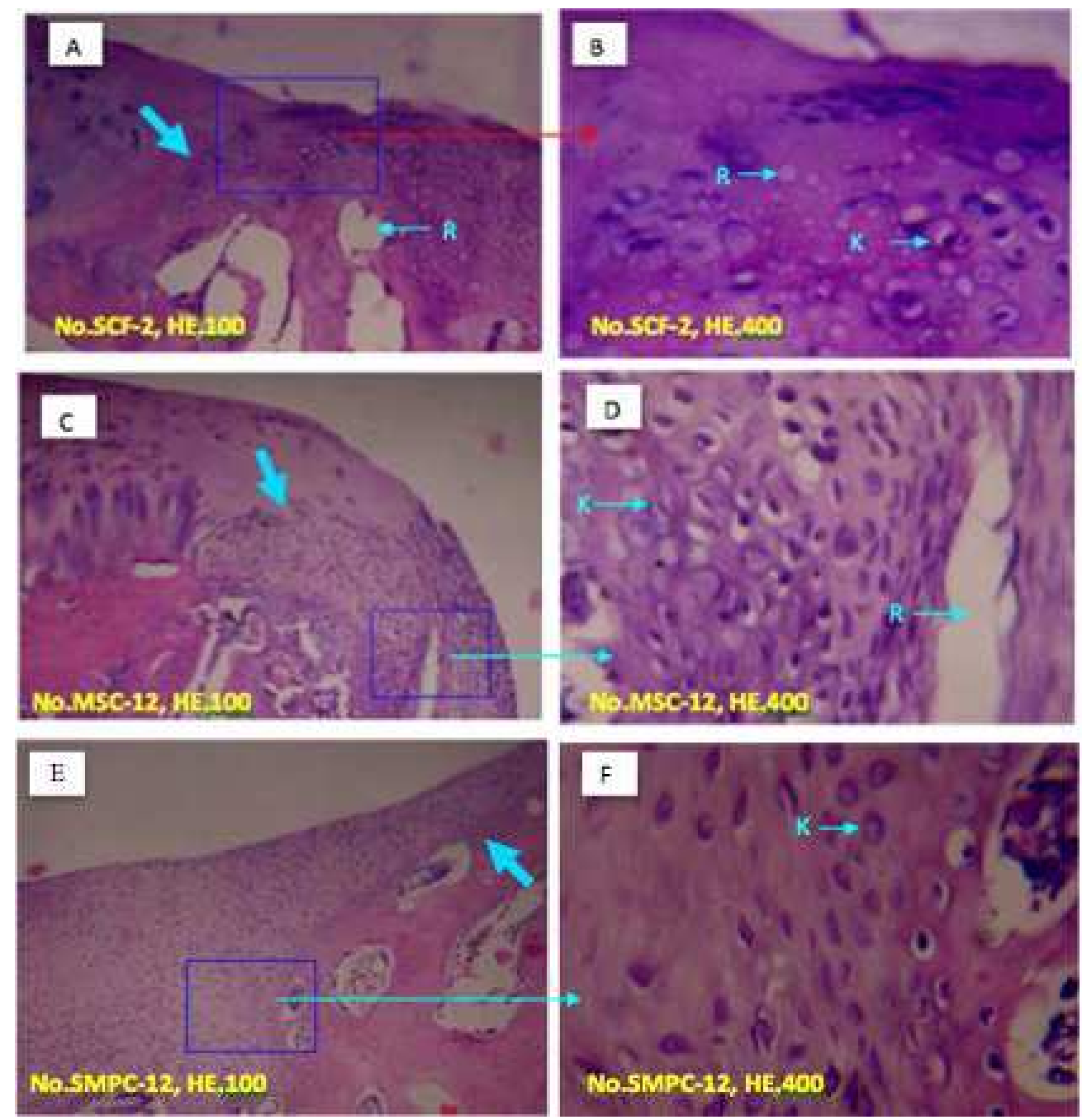

Figure 5. The number of chondrocytes in joint defect. Blue arrows show the growth of joint cartilage (condrogenesis). A: implanted with FDBC scaffold (preparation no.2, HE, x100). B: HE, x400, R: cavities; K: chondrocytes. C: implanted with MSC (preparation no.12, HE, x100) seem less horizontal surface. D: HE, x400. E: implanted with SMPC (preparation no.12, HE, x100) cartilage growth appears flat. F: shows the growth of joint cartilage homogeneous; K: condroblast (HE, x400). 

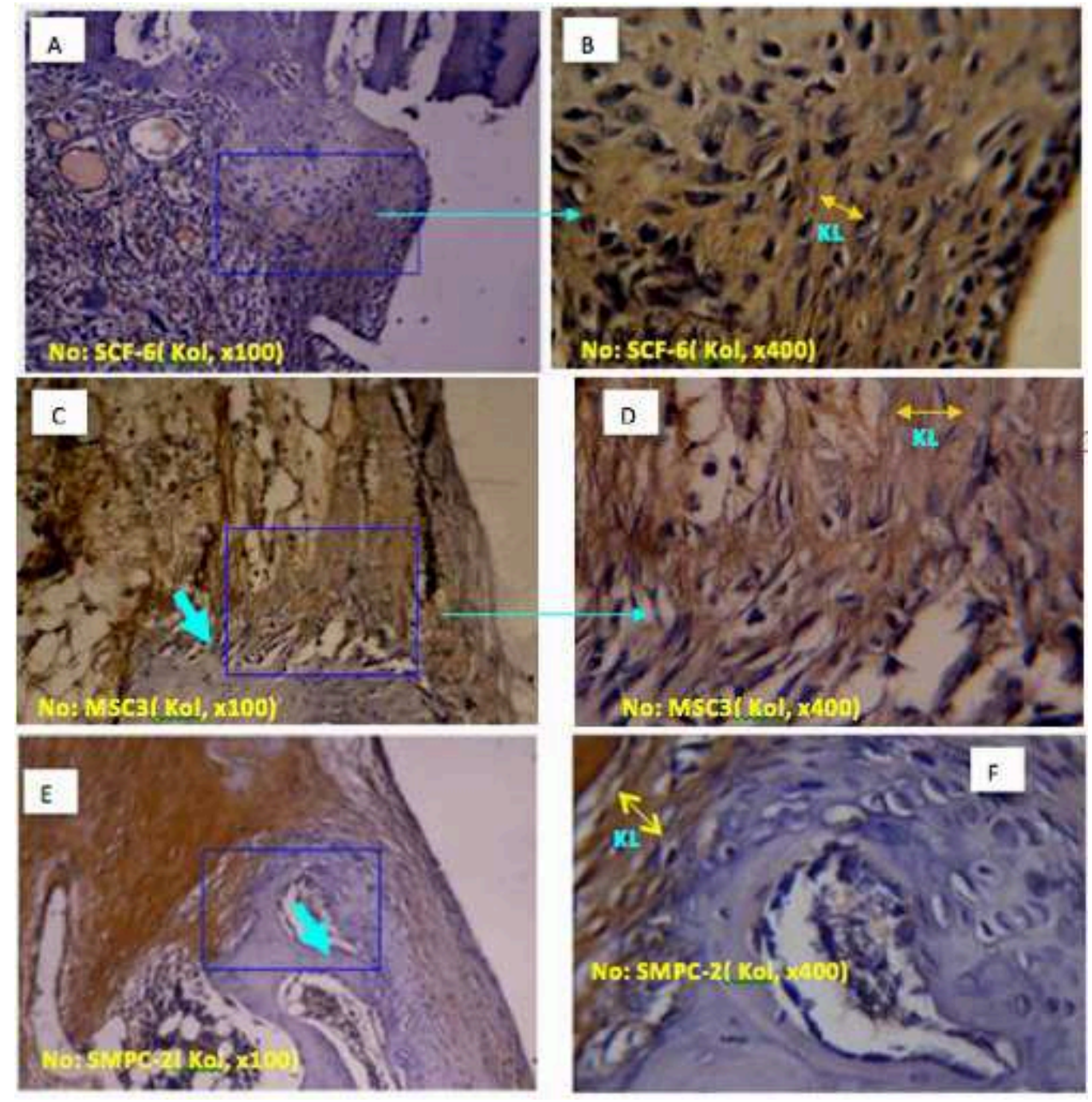

Figure 6. Collagen thickness in joint defect. Blue arrows show the growth of joint cartilage (condrogenesis). Preparation using collagen type- $2 \mathrm{mAb}$, which showed positive reaction in brown color. KL: indicates collagen thickness. A. implanted with FDBC scaffold (preparation no.6, CPI, x100) B: CPI, x400. C: implanted with MSC (preparation no.3, CPI, x100). D: CPI, x400. E: implanted with SMPC (preparation no.2, CPI, x100). F: CPI, x400. 

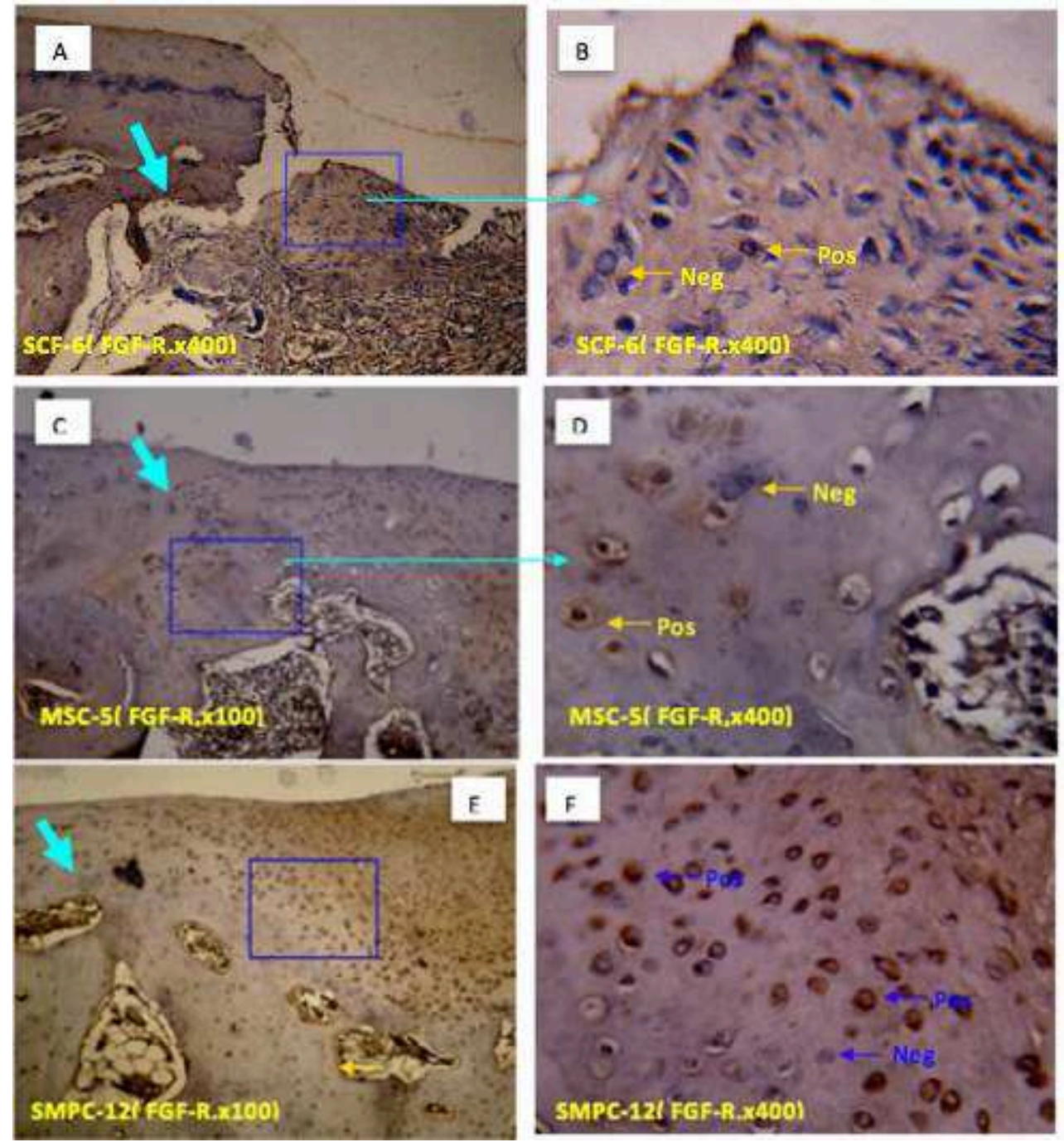

Figure 7. The number of condroprogenitor cells expressed FGF2-R in joint defect. Blue arrows show the growth of joint cartilage (condrogenesis). Preparation using mAb FGF-2R, which showed positive reaction in brown color, not brown means negative. A. implanted with FDBC scaffold (preparation no.6, CPI, x100) B: CPI, x400. C: implanted with MSC (preparation no.5, CPI, x100). D: CPI, x400. E: implanted with SMPC (preparation no.12, CPI, x100). F: CPI, x400. 

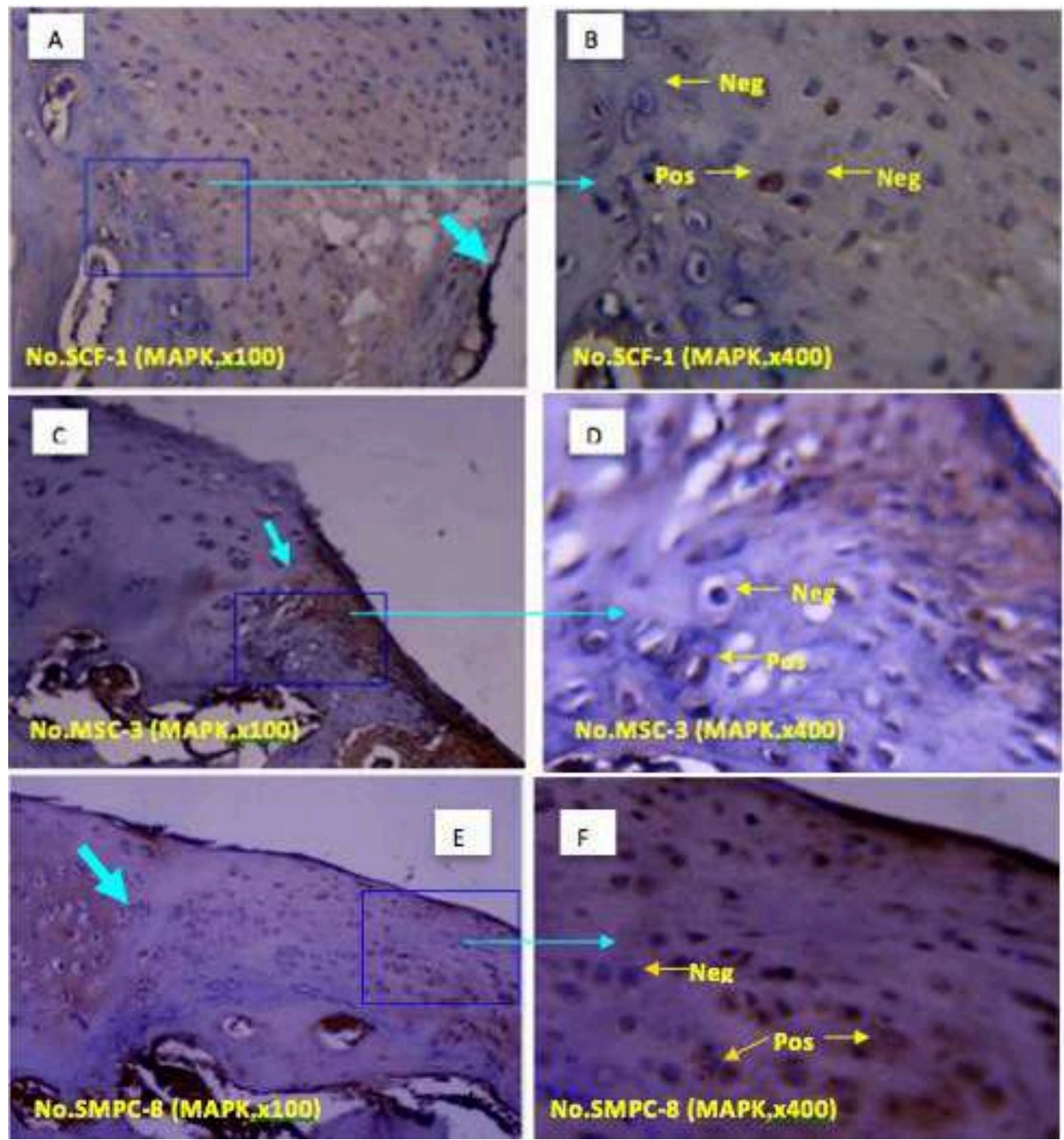

Figure 8. The number of condroprogenitor cells expressed MAPK in joint defect. Arrows show the growth of joint cartilage (condrogenesis). Preparation using mAb MAPK, which showed positive reaction in brown color, not brown means negative. A. implanted with FDBC scaffold (preparation no.6, CPI, x100) B: CPI, x400. C: implanted with MSC (preparation no.5, CPI, x100). D: CPI, x400. E: implanted with SMPC (preparation no.12, CPI, x100). F: CPI, x400. 

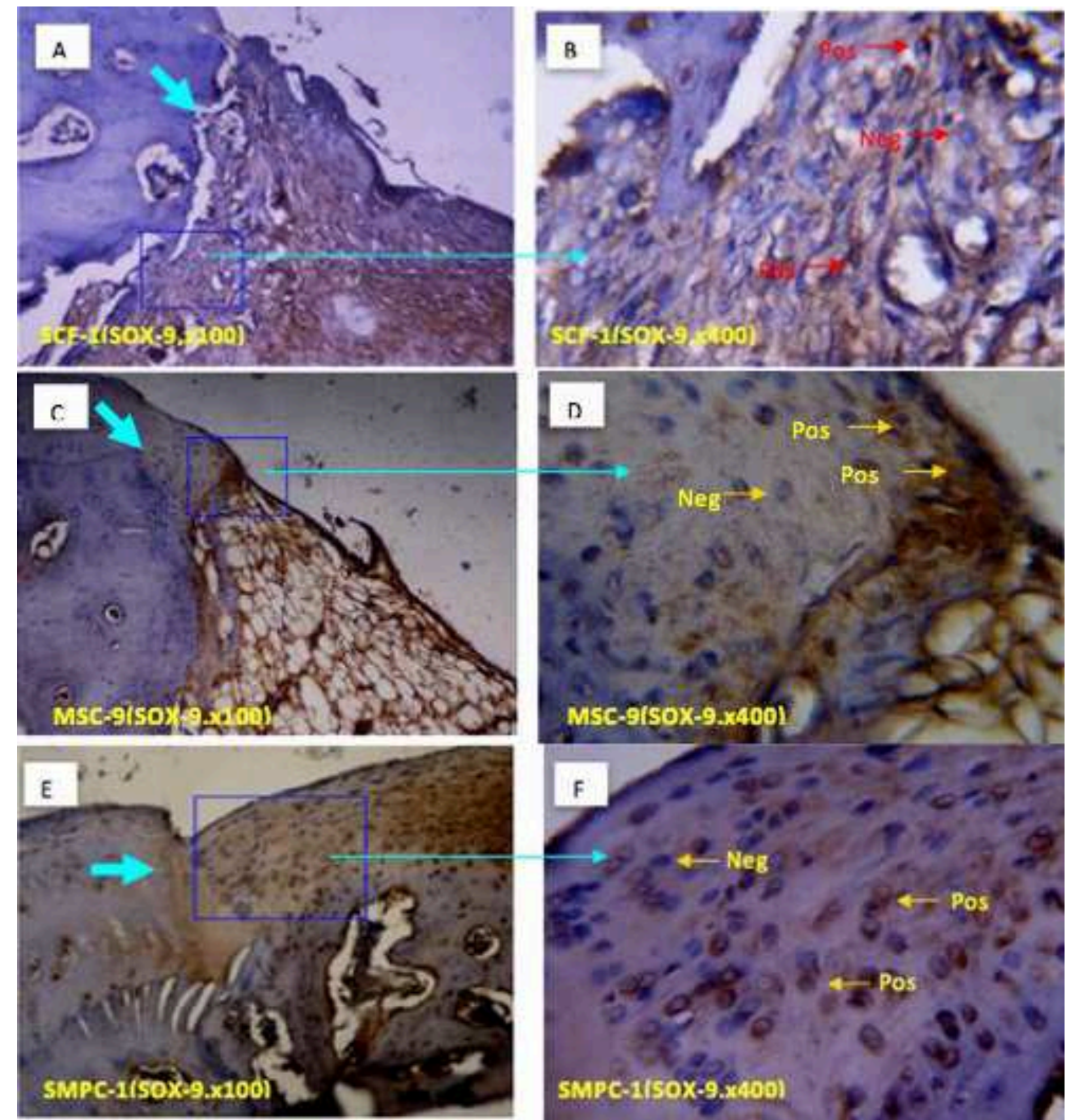

Figure 9. The number of condroprogenitor cells expressed Sox-9 in joint defect. Arrows show the growth of joint cartilage (condrogenesis). Preparation using mAb Sox-9, which showed positive reaction in brown color, not brown means negative. A. implanted with FDBC scaffold (preparation no.6, CPI, x100) B: CPI, x400. C: implanted with MSC (preparation no.5, CPI, x100). D: CPI, x400. E: implanted with SMPC (preparation no.12, CPI, x100). F: CPI, x400.

\section{References}

[1] Mononen M.E, Mikkola M.T, Julkunen P, Ojala R, Nieminen M.T, Jurvelin J.S, Korhonen R.K. 2012. Effect of Superficial Collagen Patterns and Fibrillation of Femoral Articular Cartilage on Knee Joint Mechanics-A 3D Finite Element Analysis. J Biomech. pp. 579-87.

[2] Athanasiou K.A, Shah A.R, Hernandez R.J. 2002. Basic Science of Articular Cartilage Repair. J. Clin Sport Me, 39 (4), pp. 223-47.

[3] Buckwalter J.A, Mankin H.J, Grodzinsky A.J. 2005. Articular Cartilage and Osteoarthritis. Instr Course Lect, 54, pp. 465-80.

[4] Williams III Riley J, Feeley Brian T, Bedi Askeesh. 2010. Management of Articular Cartilage Defect of The Knee. J. Bone Joint Surg Am, 92, pp. 994-1009.

[5] Chen F.H, Song Li, Mauck R.I, Li W.J. 2007. Mesenchymal Stem Cells. In: Lanza R, Langer R, Vacanti J eds. Principles of Tissue Engineering. 3rd Edition. London: Elsevier Academic Press, pp. 823-843.

[6] Getgood. 2009. Articular Cartilage Tissue Engineering: Today's Research, Tomorrow Practice? J.Bone and Joint Surgery, 91 (5), pp. 565-576. 
[7] Gikas P.D, Aston W.J, Briggs T.W. 2008. Autologous Chondrocyte Implantation: Where Do We Stand Now? J Orthop Sci, 13, pp. 283-92.

[8] Williams R.J. 2006. Articular Cartilage Repair: Clinical Approach and Decision Making. In: Williams R.J ed. Operative Tehniques in Orthopaedics. New York: Elsevier Inc, pp. 218-226.

[9] Wakitani S, Kawaguchi A, Tokuhara Y, Takaoka K. 2008. Present Status and Future Direction for Cartilage Repair. 26, Pp. 115-22.

[10] Steinert A.F, Ghivizzani S.C, Rethwilm A, Tuan R.S, Evans C.H, Noth U. 2007. Major Biological Obstacles for Persistent Cell-Based Regeneration of Articular Cartilage. Arthritis Research and Therapy, Vol 9, pp. 213-28.

[11] Koga H, Engebretsen L, Brinchmann J.E, Muneta T, Sekiya K. 2009. Mesenchymal Stem Cell-Based Therapy for Cartilage Repair: A Review. 17, pp. 1289-97.

[12] Nho S.J, Foo L.F, Green D.M. 2008. Magnetic Resonance Imaging and Clinical Evaluation of Patellar Resurfacing with Press-Fit Osteochondral Autograft Plugs. Am J Sports Med, 36 (6), pp. 1101-9.

[13] Reddi A.H. 2003. Cartilage Morphogenetic Proteins: Role in Joint Development, Homoeostasis, and Regeneration. Ann Rheum Dis, Vol 62, pp. 73-78.

[14] Ornitz D.M. 2005. FGF Signaling in The Developing Endochondral Skeleton. Cytokine \& Growth Factor Reviews, 16, pp. 205-13.

[15] Hoffman A, Stefen C, Christian K, Dietmar B, Hubert M, Yoram Z, Gadi T, Gadi P, Hou Q, DeBank P.A, Shakesheff K.M. 2004. Injectable Scaffolds for Tissue Regeneration. Journal of Materials Chemistry, 14, pp. 1915-1923.

[16] Yusbida A, Dwikora N.U. 2012. Cytotoxic Effect of Freeze Dried Bovine Scaffold and Platelet Rich Plasma on Mesenchymal Stem Cells in Cartilage Tissue Engineering. Airlangga University.

[17] Simman R, Hoffmann A, Bohinc R.J, Peterson W.C, Russ A.J. 2008. Role of Platelet-Rich Plasma in Acceleration of Bone Fracture Healing. Ann Plast Surg, 61, pp. 337-344.

[18] Shapiro F, Koide S, Glimcher. 1993. Cell Origin and Differentiation in The Repair of Full Thickness Defect of Articular Cartilage.

[19] Junji. 2009. Clinical Application of Scaffolds for Cartilage Tissue Engineering. Knee Surgeon Sports Traumatology Arthoscopy, 17, pp. 561-577.

[20] Niswander L. 2002. Interplay Between The Molecular Signals That Control Vertebrate Limb Development. The International Journal of Developmental Biology, 46, pp. 877-81.

[21] Wakitani S, Imoto K, Yamamoto T, Saito M, Murata N, Yoneda M. 2002. Human Autologous Culture Expanded Bone Marrow Mesenchymal Cell Transplantation for Repair of Artilage Defects in Osteoarthritic Knees. Osteoarthritis Cartilage. pp. 199-206.

[22] Dwikora, Sasetia D.R, Taufik A, Rofi'i, Sadabaskara, Bembie R, Yusbhida A, Adi G. 2011. Freeze Dried Bovine Cartilage Powder as An Alternative Scaffold for Cartilage Defect. International Symposia of Stem Cell and Tissue Bank, Surabaya. 